\title{
Atenção integral fragmentada a pessoa estomizada na rede de atenção à saúde
}

\author{
Fragmented comprehensive health care for ostomized person in the health care network \\ Atención integral fragmentada a la persona ostomizada en la red de atención a la salud
}

Laura Renner Bandeira ${ }^{1}$ (1) Adriane Cristina Bernat Kolankiewicz ${ }^{1}$ (1) Mariana Fröhlich Alievi ${ }^{1,2}$ (B) Letícia Flores Trindade ${ }^{1,2}$ (C) Marli Maria Loro ${ }^{1}$ (b)

${ }^{1}$ Universidade Regional do Noroeste do Estado do Rio Grande do Sul. Ijuí, RS, Brasil.

${ }^{2}$ Hospital de Caridade de ljuí. ljuí, RS, Brasil.

\section{RESUMO}

Objetivo: Identificar as ações de cuidado multiprofissional efetivadas ao estomizado do pré- operatório ao acompanhamento após a alta hospitalar. Método: Estudo descritivo de abordagem qualitativa, desenvolvido no Sul do Brasil. Incluem-se pacientes com diagnóstico médico de câncer colorretal, em uso de ileostomia ou colostomia. Coleta de dados em 2018, que se deu por meio de entrevista semiestruturada. Sortearam-se os participantes. Análise de dados de acordo com Minayo. Aspectos éticos respeitados. Resultados: Participaram 15 indivíduos. Identificou-se que os cuidados pré, pós-cirúrgico e após a alta hospitalar são fragmentados. Ainda, o estomizado encontra fragilidades no atendimento recebido na Atenção Primária à Saúde, sendo referenciado para o serviço especializado. Conclusões e implicações para a prática: $O$ indivíduo não recebe assistência adequada em sua nova condição e é no serviço especializado que são realizados cuidados que contemplam aspectos físicos e psicológicos. Os resultados podem contribuir para os profissionais e gestores em saúde, no intuito de discutir e propor ações que garantam a continuidade da atenção e a qualidade do cuidado na rede de atenção à saúde.

Palavras-chave: Estomia; Assistência integral à saúde; Atenção primária à saúde; Serviços de saúde.

\begin{abstract}
Objective: To identify the actions of multiprofessional care performed on the ostomized patient from the preoperative to the follow-up after hospital discharge. Method: Descriptive study with qualitative approach, developed in southern Brazil. Patients with a medical diagnosis of colorectal cancer who were using ileostomy or colostomy were included. Data collection was done in 2018 and through semi-structured interviews. The participants were randomly selected. Data analysis according to Minayo. Ethical aspects respected. Results: 15 individuals took part. It was identified that pre, postoperative and after discharge care are fragmented. Thus, the ostomized patient finds weaknesses in the care received in the Primary Health Care, being referenced to the specialized services. Conclusions and implications for practice: The individual does not receive adequate care in he new condition and it is in the specialized service where care is provided that the physical and psychological aspects are being cared. Results can contribute to health professionals and managers, in order to discuss and propose actions that ensure care continuity and quality in the health care network.
\end{abstract}

Keywords: Ostomy; Comprehensive health care; Primary health care; Health Services.

\section{RESUMEN}

Objetivo: Identificar las acciones de atención multidisciplinaria realizadas a los ostomizados en el período que se extiende entre el preoperatorio y el seguimiento después del alta hospitalaria. Método: Estudio descriptivo de enfoque cualitativo, desarrollado en el sur de Brasil. Se incluyeron pacientes con un diagnóstico médico de cáncer colorrectal con ileostomía o colostomía. La recolección de datos se realizó en 2018 a través de entrevistas semiestructuradas. Los participantes fueron elegidos por sorteo. Análisis de datos según Minayo. Se respetaron los aspectos éticos. Resultados: Participaron 15 personas. Se identificó que la atención recibida en el período pre y posoperatorio y después del alta es fragmentada. Además, se advierte un déficit en el servicio de salud brindado a personas ostomizadas en el ámbito de la atención primaria de salud, en lo que se refiere a servicios especializados. Conclusiones e implicaciones para la práctica: El sujeto no recibe asistencia adecuada en su nueva condición y es en el servicio especializado que se brinda la atención que abarca aspectos físicos y psicológicos. Los resultados pueden contribuir a que los profesionales y gerentes de salud logren debatir y proponer acciones que aseguren la continuidad de la atención y la calidad de la misma en la red de atención médica.

Palabras clave: Ostomía; Atención integral de salud; Atención primaria de salud; Servicios de Salud.
Autor correspondente:

Adriane Cristina Bernat Kolankiewicz E-mail: adri.saudecoletiva@gmail.com

Recebido em 04/11/2019.

Aprovado em 09/02/2020.

DOI:

https://doi.org/10.1590/2177-9465-EAN-2019-0297 


\section{INTRODUÇÃO}

Na contemporaneidade, o câncer intestinal contribui para o aumento de procedimentos cirúrgicos abdominais, dentre eles, a colostomia e ileostomia. Estimativa do Instituto Nacional de Câncer ${ }^{1}$ prevê 36.360 novos casos de câncer colorretal (CCR), sendo 17.380 em homens e 18.980 em mulheres. Esta patologia corresponde a $36 \%$ das razões mais frequentes para a realização de cirurgia de estoma intestinal. ${ }^{2}$

O termo "ostomia" origina-se do grego stómae, que significa abertura e exteriorização de uma víscera oca, por meio do ato cirúrgico. Estas podem ser temporárias, ou definitivas, a depender da possibilidade, ou não, de reconstruir o trânsito intestinal. ${ }^{3}$

Nesse sentido, esta condição demanda dos serviços de saúde atendimento articulado e multiprofissional. Fato que pode estar relacionado às distintas necessidades do estomizado, com vistas à adaptação à nova condição. ${ }^{4} \mathrm{~A}$ fim de garantir que $\mathrm{O}$ paciente tenha sua autonomia e adaptação estabelecidas, cabe aos profissionais de saúde realizarem ações educativas, como as orientações de cuidados no pré-operatório e, em especial, no pós-operatório, pois o paciente e familiar necessitam de informações que lhes deem capacidade de cuidar da estomia no domicílio. A efetivação destas ações é fundamental para a reabilitação do paciente e o cuidado em domicílio. Entretanto, autores inferem que isto ocorre parcialmente, o que compromete a assistência. ${ }^{5}$

Para além disso, após a alta hospitalar, a Atenção Primária à Saúde (APS), bem como o serviço especializado, que compõem a RAS, devem realizar assistência planejada e contemplar aspectos fisiológicos e psicológicos do paciente e sua família, na perspectiva de garantir a integralidade de saúde. ${ }^{6}$ Para tanto, é primordial que todos os profissionais participem efetivamente do processo de cuidado, a fim de garantir sua continuidade e a assistência integral à saúde. ${ }^{7}$ Contudo, evidências pontuam falhas de comunicação entre os níveis primário e secundário de atenção à saúde. ${ }^{8}$

Entre as fragilidades, observa-se que os serviços de saúde atuam de forma articulada na rede de atenção ainda em um processo incipiente, o que resulta em um cuidado fragmentado, principalmente, quando do cuidado ao estomizado. Destaca-se a necessidade do fortalecimento da RAS, que visa à integralidade no cuidado, o que permitirá assistência contínua, respondendo às necessidades e particularidades de cada sujeito. Outro aspecto refere-se, ainda, à incipiência de estudos acerca da temática e deste grupo populacional que discutam a atenção à saúde ofertada a estes sujeitos na RAS. Tendo em vista tais aspectos, estabeleceu-se como pergunta de pesquisa: Quais cuidados são efetivados ao paciente estomizado na RAS? E, como objetivo do estudo, identificar as ações de cuidado multiprofissional efetivadas ao estomizado do pré-operatório ao acompanhamento após a alta hospitalar.

\section{MÉTODO}

Trata-se de um estudo descritivo, de abordagem qualitativa, que integra uma dissertação de mestrado intitulada "Saberes e práticas de cuidado ao estomizado na rede de atenção à saúde", desenvolvido em um município do Sul do Brasil, com população estimada de 83.173 pessoas.

Para estabelecer a população participante, solicitou-se junto à Secretaria Municipal de Saúde a relação nominal de indivíduos cadastrados no Sistema de Gerenciamento de Usuários com Deficiência (GUD) com diagnóstico de CCR, onde obtiveram-se 29 usuários. Tem-se como critérios de inclusão: maiores de 18 anos, com diagnóstico médico de CCR, em uso de ileostomia ou colostomia. Sortearam-se os indivíduos para participarem do estudo, com reposição de participantes caso houvesse recusa, deste modo, obtiveram-se 15 participantes.

A coleta de dados ocorreu durante o primeiro semestre de 2018, por meio de entrevista semiestruturada (conduzidas pela primeira e terceira autora). A mesma contempla questões fechadas que possibilitaram caracterizar os participantes do estudo e questões abertas sobre o tema em tela, as quais permitiram o diálogo e reflexão sobre a temática estudada.

As entrevistas foram realizadas no domicílio, mediante agendamento prévio por contato telefônico, em que a data e horário das mesmas foram estabelecidos pelo participante. Importante salientar as dificuldades encontradas nesta etapa, devido a endereços incompatíveis, óbitos recentes de participantes, dificuldades de acesso ao domicílio, etc.

Primeiramente, sugeriu-se ao participante que a conversa fosse realizada em local tranquilo e privado, a fim de que este pudesse explanar sobre sua condição. Em seguida, esclareceu-se sobre o objetivo do estudo e, após, explicou-se que a participação possuía caráter voluntário e que poderia ser interrompida a qualquer tempo. Quando se obteve o aceite do participante, o mesmo assinou o Termo de Consentimento Livre Esclarecido (TCLE) em duas vias, sendo uma via do participante do estudo e a outra, do pesquisador.

Sequencialmente, iniciaram-se as entrevistas, perdurando um tempo médio de 15 minutos, sendo gravadas e transcritas na íntegra. Com vistas a garantir o anonimato, os participantes do estudo foram identificados pela letra $p$, seguida do número sequencial correspondente à entrevista, ou seja, de P1 a P15.

A análise dos dados foi realizada como preconizado por Minayo $^{9}$, em três etapas: ordenação dos dados por meio da transcrição das entrevistas gravadas, leitura, organização das entrevistas e identificação das temáticas; classificação dos dados a partir da releitura dos textos e material obtido, para identificar as temáticas comuns e, posteriormente, agrupá-las em categorias de acordo com o tema; e análise final dos dados, considerando-se a base teórica e relacionando-se os dados referentes à observação simples dos dados, reportando-se aos objetivos propostos.

Os aspectos éticos foram respeitados de acordo com a Resolução do Conselho Nacional de Saúde 466/2012 ${ }^{10}$, e o projeto foi aprovado pelo Comitê de Ética em Pesquisa (CEP), sob CAAE 80479417.2.0000.5322.

\section{RESULTADOS}

O estudo incluiu 14 indivíduos que possuíam colostomia intestinal e um com ileostomia, mas todos apresentavam estoma definitivo. Destes, $80,1 \%$ (12) encontravam-se na faixa etária 
entre 51 e 80 anos, 53,3\% (8) eram do sexo feminino, 40\% (6), viúvos e 33, (5) casados. Quanto à ocupação, 46,7\% (7) eram aposentados, e $53 \%$ (8) tinham ensino fundamental incompleto. Ainda, 86,7\% (13) dos respondentes possuíam estomia de um a cinco anos.

A análise dos depoimentos permitiu a identificação de duas categorias de análise: Cuidados multiprofissionais efetivados ao estomizado do pré operatório ao acompanhamento após a alta hospitalar; Itinerário terapêutico e centralização do cuidado ao estomizado.

Cuidados multiprofissionais efetivados ao estomizado do pré operatório ao acompanhamento após a alta hospitalar

Um procedimento cirúrgico é constituído por várias etapas, sendo denominadas como pré, trans e pós-operatório. Ele tem seu início estabelecido após a avaliação da indicação do procedimento, quando se obtém o consentimento, e inicia-se a preparação cirúrgica. Constata-se que as orientações no período pré-operatório são fundamentais, porém se observa que muitas vezes elas não ocorrem, como evidencia-se nos relatos:

Não fui orientado antes do procedimento (P5, P6, P10, P12, P15).

Não fui orientada, nunca ninguém me disse nada, não tinha nem noção. $E$ isso é bem importante, porque eu nunca passei por isso e a gente precisa ser orientada e (que) nos (seja) explicado o que será feito (P13).

Nesse sentido, as orientações visam esclarecer os motivos que levaram à necessidade da confecção do estoma, se este é temporário ou definitivo, suas possíveis complicações e os cuidados que se deve ter no manuseio e manutenção do mesmo.

Ainda destaca-se que, em maior número, os participantes do estudo referiram ter recebido orientações da equipe multiprofissional, conforme relatos abaixo:

Antes do procedimento fui orientado pelo médico (P2).

[...] fui orientado lá no hospital, era da enfermagem (P3, P8, P14, P9).

Fui orientada pelo médico, psicólogo, e pela enfermagem lá no hospital (P7, P1).

Logo, observa-se a importância das orientações cirúrgicas, com vistas a esclarecer dúvidas e facilitar o processo de aceitação/adaptação. Para obter-se assistência humanizada e qualificada é necessária a participação de diferentes profissionais. As orientações devem ser realizadas independente do profissional atuante, logo, não estão vinculadas a uma classe profissional. No entanto, é importante ressaltar que o enfermeiro, dentre todas as categorias profissionais, é o que permanece maior tempo com o paciente.

Após o procedimento a equipe de enfermagem me ajudou e orientou (P8).
Depois que fiz a cirurgia, sim, me falaram, orientaram a minha filha (P9).

A realização das orientações requer competência profissional, assim como conhecimento, habilidades e atitudes para a realização desta ação. Para além disso, as ações devem ser individualizadas e considerar o conhecimento prévio do indivíduo, de forma a responder às dúvidas e atender as necessidades de cada paciente. Tal aspecto também foi mencionado em relato de participante do estudo:

Trocaram a bolsa e me mostraram, mas eu não estava entendendo nada, não conseguia assimilar nada daquilo ali, ninguém me falou quando tinha que limpar, trocar, cortar aquilo (P12).

Além das orientações pré-operatórias, as de pós-operatório são, também, decisivas para o autocuidado, aceitação e qualidade de vida. Sendo assim, os profissionais de saúde precisam estar presentes no pós-operatório, a ofertar suporte constante. Neste sentido, participantes do estudo expressaram orientações recebidas no pós-cirúrgico:

Fui orientada quanto à higienização, que eu tinha que controlar o que comia, daí iria controlar o meu intestino, [...] como trocava a bolsa [...] (P10, P11).

Me explicaram o que podia acontecer, para que servia [...] mostraram bastante coisa da bolsa, como que tinha que fazer para lavar e esvaziar, [...] que as trocas eram a cada 7 dias $[. .].(P 2, P 3 P 8, P 13, P 14)$.

Torna-se importante envolver os familiares, pois em muitas situações serão estes que realizarão os primeiros cuidados no domicílio, como a troca da bolsa, limpeza, entre outros cuidados, desde modo, precisam também estar aptos para prestá-los. Fato que também é identificado nos depoimentos dos participantes:

Eles falaram para meu familiar como limpar, trocar a bolsa, usar todos os produtos, como recortar, mostraram os modelos (P4, P5).

Minha filha foi orientada no hospital, eu não sei dizer (P9).

Ademais, o repasse do conhecimento ao estomizado e seus familiares pode ser realizado de inúmeras formas, as quais facilitam a apreensão das informações. O uso de cartilhas, guias, folders influencia positivamente na aprendizagem, método que também foi identificado na fala de participante:

Me explicaram, me deram um papel e leram dizendo como limpar e trocar $(P 7)$.

Todas estas ações favorecem o trabalho em equipe na medida em que contribuem para a assistência em saúde qualificada e a padronizam. 


\section{Itinerário terapêutico e centralização do cuidado ao estomizado}

Os cuidados com os pacientes com CCR iniciam-se no diagnóstico e necessitam de uma equipe profissional capacitada, para prepará-los para um provável procedimento cirúrgico, ou outro de tipo de tratamento, e este deve iniciar na APS. Haja vista que estão adscritos a esta unidade, que a equipe multiprofissional conhece seu processo saúde/doença e pelo vínculo que o paciente tem com os profissionais da unidade. Infere-se, ainda que, mesmo o paciente sendo encaminhado a outros pontos da RAS, como uma instituição hospitalar, este cuidado deve ser coordenado pela APS.

Nesse sentido, após a confecção da estomia e sua alta hospitalar, a adaptação do estomizado inicia-se no domicílio. É potencializada após perceber-se em casa, sem profissionais de saúde para realizar os cuidados. Assim, antes da alta hospitalar os profissionais da equipe multiprofissional devem referenciar os pacientes e familiares para a APS, com vistas à continuidade do cuidado. Contudo, os participantes revelaram em seus depoimentos fragilidades na contrarreferência, como se observa nos relatos.

Não fui encaminhado para a unidade de referência do bairro (P1, P2, P3, P4, P6, P7, P8, P9).

Fui orientado para ir na unidade referência para pegar as bolsas de colostomia (P10, P11, P12, P14, P15).

Destaca-se, que o nível secundário de assistência deve realizar a contrarreferência para a APS, pois a comunicação efetiva entre os distintos níveis de atenção é importante para a reabilitação do paciente. Porém, a fragilidade está explícita nos relatos de alguns dos participantes do estudo.

Não tive nenhum acompanhamento da unidade de saúde de referência $(P 1)$.

A minha esposa procurou, mas não quiseram me atender naquele dia (P3).

Só procuro a unidade de saúde para outras coisas, mas não para receber atendimento para a colostomia (P14).

A APS é a porta de entrada dos serviços de saúde e a ordenadora do cuidado na RAS. Contudo, participantes do estudo vivenciaram dificuldades explicitadas em seus relatos:

[...] a enfermeira falou à minha esposa o que era a ostomia, mas não sabiam quase nada [...] (P1).

Só procuro a unidade de saúde para outras coisas, mas não para receber atendimento para a colostomia, porque eles não sabem (P14).

Estou cadastrado no ESF do bairro, mas vou no posto central, porque aqui na unidade ninguém sabe disso (P7).
Nesse sentido, destaca-se que, no município em que foi realizado o estudo, há um espaço específico que realiza atendimento aos pacientes estomizados. Neste, atuam uma enfermeira estomaterapeuta e técnicos de enfermagem. Estes profissionais realizam o cadastro dos estomizados em um sistema específico, para que os pacientes recebam, gratuitamente, os materiais de cuidado do estoma, além de realizar o cuidado com o estoma (pele, dispositivos, nutrição, apoio psicológico), como preconizam as Diretrizes Nacionais para a Atenção à Saúde das Pessoas Estomizadas.

Contudo, isso não desresponsabiliza os profissionais de saúde da unidade em que o usuário é adscrito de assisti-los. Ademais, a assistência efetivada na APS deve ser resolutiva nas demandas dos indivíduos em seu domicílio e, quando necessário, a equipe da APS pode buscar suporte na equipe especializada, no entanto, deve haver comunicação entre os serviços. Porém, como observado nos relatos:

Não tive nenhum acompanhamento da unidade de saúde de referência, somente os profissionais da unidade do centro vieram duas vezes ( $P 1)$.

O médico disse que qualquer dúvida era lá na unidade central que iam ajudar [...] (P12).

Fui para a unidade do bairro, mas me orientaram a procurar a unidade do centro, porque minha bolsa tinha soltado e eu estava com diarreia, aí me encaminharam para lá, dizendo que ali eles não sabiam mexer nisso (P14).

O maior percentual dos entrevistados apontou que só recebeu orientação e cuidados adequados/especializados quando entrou em contato com serviço especializado. Isso foi evidenciado nos relatos dos participantes deste estudo:

Recebi orientação, fui muito bem tratado no serviço especializado (P2).

Na unidade central foi 100\%! Lá que aprendi tudo (P8).

A enfermeira do serviço especializado me mostrou como limpar e trocar a bolsinha, me deu uns papéis, e agora vai tranquilo. Depois que enfermeira me ensinou, não tive mais problema (P7).

A enfermeira me mostrou como limpar e trocar a bolsinha, me deu uns papéis, e agora vai tranquilo. Depois que ela me ensinou, não tive mais problema (P14).

Para tanto, uma das estratégias realizadas pela unidade de referência é o grupo de estomizados. Tais aspectos estão explicitados nos depoimentos:

[...] comecei a ir no grupo, então me orientaram algumas coisas de alimentação (P15).

[...] quando fui retirar as bolsas no centro, fiquei sabendo de um grupo de estomizados, então comecei a participar (P15). 
Desse modo, percebe-se o grupo como intervenção efetiva e necessária para a consolidação do autocuidado do estomizado, na medida em que são disponibilizadas informações sobre alimentação, cuidados com o estoma, além de permitir o convívio social. No entanto, a ocorrência de grupos e de um centro especializado não exime a responsabilidade da APS frente a esses indivíduos.

\section{DISCUSSÃO}

O CCR abrange tumores do intestino grosso e do reto, e a maioria deles acomete o reto e ânus. Nesses casos, a cirurgia é a opção de tratamento e manutenção da vida, a qual resulta, na maioria das vezes, na confecção de um estoma intestinal. ${ }^{11}$

Contudo, observa-se que, por vezes, a realização da intervenção cirúrgica pode ocasionar experiências negativas, emocionalmente e fisicamente, em especial nas cirurgias em que há confecção de um estoma intestinal. Assim, muitos indivíduos apresentam sentimentos de ansiedade ou medo em decorrência do desconhecido, da anestesia, da mutilação e, até mesmo, da possibilidade de morte, ao se submeterem ao procedimento. ${ }^{7}$

Nesse sentido, o acompanhamento desse paciente na APS poderá contribuir na amenização desses sentimentos, pois, a partir do apoio recebido da equipe, o paciente pode ficar mais bem preparado para o enfrentamento desta nova condição. Deste modo, é fundamental que a equipe multiprofissional da APS auxilie no processo de enfrentamento da doença e fases subsequentes.

De acordo com autores ${ }^{12}$, a fase pré-operatória compreende o momento da tomada de decisão do paciente de submeter-se ao procedimento, até a sua transferência para a mesa cirúrgica. Nela, os profissionais da equipe multiprofissional devem realizar ações educativas, com vistas a esclarecer dúvidas sobre a intervenção, reduzir a ansiedade, o estresse e medos que a intervenção cirúrgica impõe. ${ }^{5}$

Além disso, as orientações nesta fase contribuem, positivamente, para evitar complicações futuras, com vistas à recuperação do paciente, sua qualidade de vida, além de contribuir para a aceitação da mudança corporal, e colaboram para a interação profissional-paciente. ${ }^{12}$

Destaca-se que um número considerável de participantes desta pesquisa relatou não ter recebido orientações. E estudo 5 corrobora com os resultados do estudo em tela, na medida em que destacou que muitos dos participantes não receberam orientações e/ou não se recordavam se haviam sido orientados.

Quando o paciente não recebe orientações no pré-operatório, fica vulnerável a alterações significativas e a intervenção realizada pode ser percebida como traumatizante, uma vez que o indivíduo se encontra abalado pelas informações acerca do diagnóstico, da intervenção cirúrgica e da construção de um estoma. ${ }^{13}$

Ademais, quanto maior o estresse e a tensão no pré-cirúrgico, maiores serão as dificuldades para enfrentar os limites que a cirurgia impuser ao cotidiano do paciente. ${ }^{7} \mathrm{Em}$ relação aos cuidados com o estoma, autor ${ }^{14}$ pontua que é importante mostrar imagens e estomas intestinais com e sem bolsa coletora, permitir que o paciente e sua família manipulem os materiais de que farão uso, ofertar o contato direto e real com os materiais, para facilitar a aceitação da nova condição.

Desse modo, as ações educativas efetivadas têm influência positiva nas técnicas de autocuidado, na aceitação da nova condição e adaptação à mesma. Na mesma medida, permitem que pacientes aprendam a cuidar-se, sintam-se seguros diante da situação e passem a compreender melhor o que estão vivenciando. ${ }^{15}$

A interação multiprofissional é necessária e fundamental, pois incentiva o trabalho integrado com vistas ao bem-estar do usuário e propicia a troca de saberes, a articulação das ações e a integração entre os membros da equipe. ${ }^{16}$

Em estudo ${ }^{11}$, os entrevistados não mencionaram ter recebido orientação de enfermeiro, relacionaram-na ao médico, e foram direcionadas ao procedimento cirúrgico, excluindo-se orientações de autocuidado. Ademais, em outro estudo, 75\% dos depoentes afirmaram que receberam orientação no período pré-operatório, sendo a maioria pelos enfermeiros. ${ }^{5}$

O enfermeiro está presente desde o diagnóstico, antes, durante e após o procedimento cirúrgico, bem como na reabilitação. Portanto, cabe a ele realizar ações educativas que visem à autonomia do paciente, pois ele é responsável pelas orientações de autocuidado, principalmente relacionado ao manuseio do estoma e do equipamento coletor. ${ }^{15}$

Autores $^{4}$ corroboram, ao inferirem que as ações de ensino-aprendizagem são função do enfermeiro e permitem o autocuidado e autonomia, consequentemente, colaboram para a qualidade de vida, convívio com seus familiares e a reinserção social, ações que podem ser identificadas nos depoimentos.

As orientações devem se dar de forma contínua e sistematizada, pois mostram-se efetivas para a aquisição e compartilhamento de informações, além de serem uma ferramenta essencial para obter assistência de qualidade. ${ }^{5} \mathrm{O}$ momento após a cirurgia deve ser trabalhado de forma específica e utilizando metodologias que garantam a compreensão e o envolvimento do sujeito no autocuidado. ${ }^{17}$

Nessa fase, as orientações devem centrar-se nos cuidados com a pele do peristoma e troca de bolsas coletoras, quanto aos hábitos alimentares, higiene do estoma e do dispositivo coletor, além de estimular o autocuidado, que influencia, positivamente, no processo de adaptação ao estoma e previne complicações. ${ }^{18}$

Importante salientar que, durante esse processo de aprendizagem, deve-se incluir a família. Ela intervém como uma importante rede de apoio, na medida em que atua como mediadora para enfrentar a situação vivenciada antes e após a alta hospitalar, exercendo o cuidado no domicílio e ao fornecer suporte emocional. ${ }^{2,4}$

O cuidador familiar, além do cuidado com o estoma, é responsável pelo apoio emocional, por meio de diálogos, escutas, explicações, conselhos, conferindo conforto e segurança, diminuindo medos e angústias da pessoa com estomia intestinal. ${ }^{2,4}$

Nessa perspectiva, a utilização de materiais educativos é um mediador entre o profissional e o paciente/familiar, pois, além de auxiliarem a assimilar as orientações por meio de textos e 
figuras, também representam um recurso disponível para que possam consultá-lo no momento de dúvidas. ${ }^{19}$

A partir da intervenção cirúrgica e da alta hospitalar, o indivíduo terá o desafio de adquirir habilidades para conviver com o corpo alterado e experimentará uma transição psicossocial. ${ }^{7}$ Neste sentido, o estomizado conviverá com mudanças significativas na vida, devido às alterações físicas, que interferem diretamente no seu estilo de vida e exigem adaptação à sua nova condição. ${ }^{2}$

Importante que ocorra a contrarreferência destes pacientes, no intuito de as equipes de saúde da família darem a continuidade do cuidado, as quais devem atender às demandas em saúde dos usuários adscritos a um território definido, com vistas a desenvolver atenção integral, de modo que impacte, positivamente, na situação de saúde e autonomia dos indivíduos. ${ }^{6}$

Contudo, de acordo com autores ${ }^{8}$, existem dificuldades na continuidade da assistência determinadas pela inexistência de comunicação entre os sistemas de cuidado. Os mesmos autores ${ }^{8}$ enfatizam a necessidade diálogo entre a equipe dos níveis de atenção primário e secundário, com o paciente e sua família, a fim de garantir o cuidado integral e continuado no domicílio.

Destaca-se que a APS é considerada a coordenadora do cuidado, com o papel de ordenar a RAS, além disso, tem como propósito facilitar o acesso da população e é a porta de entrada dos serviços de saúde, assim, cabe aos profissionais atuantes na mesma efetivar ações de promoção, prevenção, proteção, diagnóstico, tratamento, recuperação e reabilitação da saúde ${ }^{6}$

Em estudo ${ }^{20}$ realizado com 16 enfermeiros da APS, constatou-se que seis deles tinham conhecimento incipiente sobre ileostomia e dois desconheciam a temática, o que denota déficit na formação deste profissional. Tais achados vão ao encontro de estudo ${ }^{21}$ que identificou insegurança e respostas inadequadas quanto à descrição do procedimento da troca do equipamento, evidenciando a necessidade de que os enfermeiros se apoderem desta temática.

Assim, diante dessas fragilidades, percebe-se que 0 estomizado não é assistido integralmente pela equipe na APS, o que faz com que este indivíduo fique desassistido na RAS. Nessa perspectiva, as Diretrizes Nacionais para a Atenção à Saúde das Pessoas Estomizadas ${ }^{22}$ garantem a atenção integral à saúde da pessoa com estoma, o que inclui o fornecimento de materiais que favoreçam o autocuidado.

A descontinuidade e desarticulação das redes de atenção permitem a centralização do cuidado e não permitem a assistência integral à saúde ${ }^{23}$. Estudo infere que, quando o paciente estomizado chega ao centro de referência, já percorreu vários pontos da RAS, fato justificado pela assistência ineficaz, ocorrência de situações frustrantes que explicitam a fragilidade atual, representadas pelas vivências dos estomizados que buscam nos profissionais respostas para as suas angústias e, em alguns momentos, defrontam-se com a precariedade de informações, bem como de cuidado. ${ }^{23}$

A maioria dos usuários estomizados só recebe orientação e cuidados adequados quando entra em contato com serviço especializado. ${ }^{24} \mathrm{~A}$ assistência especializada às pessoas com estoma objetiva a reabilitação, com ênfase na orientação para o autocuidado, ao desenvolver habilidades para a realização de suas atividades de vida diária, prevenção de complicações nas estomias e fornecimento de equipamentos coletores e adjuvantes de proteção e segurança. ${ }^{25}$

O grupo de apoio proporciona interação entre os participantes, contribui para o compartilhamento de experiências e favorece o convívio social. A convivência com outras pessoas estomizadas é um método de adquirir conhecimentos por meio de trocas de experiências, pois cada participante enfrenta as adversidades com um olhar diferenciado e, no momento em que essas experiências são trocadas, o elo de confiança se fortalece, fazendo com que haja motivação para o enfrentamento dos desafios. ${ }^{18}$

Autore ${ }^{18}$ pontuam que esta estratégia educacional oportuniza o desvendamento de vários desafios, trazendo à tona novos olhares para a sua condição física, permite maior autonomia e participação social. O paciente ostomizado requer cuidados diversos e estes repercutem em sua vida. Para tanto, a atenção da equipe multiprofissional é importante em todo seu itinerário terapêutico ${ }^{26}$, cuidados que visam garantir a integralidade do cuidado na RAS.

\section{CONSIDERAÇÕES FINAIS E IMPLICAÇÕES PARA A PRÁTICA}

Resultados permitem inferir que as ações de cuidado ofertadas no pré e pós-operatório são incipientes, bem como o acompanhamento destes pacientes após a alta hospitalar, o que fragiliza o cuidado integral, esperado na RAS. Ao acessar sua UBS/ESF de referência, os estomizados são referenciados e orientados a buscar cuidados no serviço especializado.

Esse serviço deve ser entendido como suporte à assistência efetivada na unidade de saúde em que o usuário é adscrito, contudo, é ele que efetiva o cuidado físico e psicológico a esses usuários e seus familiares. Nesse sentido, é importante que a equipe multiprofissional das UBS/ESF se apodere de conhecimentos para dar conta das necessidades dos indivíduos estomizados por meio de ações de cuidado continuadas, no intuito de desenvolver habilidades e conhecimentos.

Nessa perspectiva, sugere-se aos profissionais de saúde e gestores que repensem seu processo de trabalho, com vistas a responder às necessidades de cuidado dos usuários, haja vista que a UBS/ESF deve ser o acesso prioritário e deve resolver a maioria dos problemas da população adscrita. Ainda, é necessária a efetivação de ações de educação permanente no intuito de potencializar práticas assistenciais e de gestão do cuidado para responder às demandas advindas dos estomizados e seus familiares.

A consolidação do cuidado integral aos estomizados requer ações de apoio matricial pela equipe do serviço especializado para as equipes da APS, com vistas a ofertar suporte técnico específico às equipes multiprofissionais, o que qualificará o cuidado prestado aos estomizados. 


\section{FINANCIAMENTO}

Conselho Nacional de Desenvolvimento Científico e Tecnológico (CNPQ). Programa Institucional de Bolsas de Iniciação Científica (PIBIC). Bolsa de iniciação científica, concedida a Laura Renner Bandeira e Letícia Flores Trindade no período de 01 de janeiro a 31 de dezembro de 2018

\section{CONTRIBUIÇÕES DOS AUTORES}

Concepção e desenho do estudo. Coleta de dados. Análise e interpretação dos resultados. Redação e revisão crítica do manuscrito. Aprovação da versão final do artigo. Responsabilidade por todos os aspectos do conteúdo e a integridade do artigo publicado. Laura Renner Bandeira e Mariana Frölich Alievi

Concepção e desenho do estudo. Análise e interpretação dos resultados. Redação e revisão crítica do manuscrito. Aprovação da versão final do artigo. Responsabilidade por todos os aspectos do conteúdo e a integridade do artigo publicado. Adriane Cristina Bernat Kolankiewicz, Letícia Flores Trindade e Marli Maria Loro

\section{EDITOR ASSOCIADO}

\section{Maria Catarina Salvador da Motta}

\section{REFERÊNCIAS}

1. Instituto Nacional do Câncer. Câncer de intestino [Internet]. Brasília INCA; 2018 [citado 2018 dez 1]. Disponível em: https://www.inca.gov. br/tipos-de-cancer/cancer-de-intestino

2. Pinto IMS. Desenvolvimento da competência de autocuidado da pessoa com ostomia de eliminação intestinal: validação do formulário [dissertação]. Porto: Escola Superior de Enfermagem do Porto; 2014 [citado $2018 \mathrm{dez}$ 1]. Disponível em: https://comum.rcaap.pt/bitstream/10400.26/9537/1/ Igor_Pinto_Dissertacao_Mestrado_Porto_2014.pdf

3. Ribeiro RVL, Oliveira AC, Viana LV, Pinto AP, Carvalho ML, Elias CMV Adaptação social do paciente colostomizado: desafios na assistência de enfermagem. Rev. Interd. 2016;9(2):216-22.

4. Ardigo FS, Amante LN. Knowledge of the professional about nursing care of people with ostomies and their families. Texto Contexto Enferm. 2013 dez;22(4):1064-71. http://dx.doi.org/10.1590/S0104-07072013000400024.

5. Silva RM, Soares RSA, Lana LD, Birrer JA, Mostardeiro SCTS. Orientações pré-operatórias recebidas por pacientes submetidos à cirurgia oncológica. Rev. Cient. Sena Aires [Internet]. 2017; [citado 2018 dez 1];6(2):116-25. Disponível em: http://revistafacesa.senaaires.com. br/index.php/revisa/article/view/286

6. Portaria $\mathrm{n}^{\circ} \mathbf{2 . 4 3 6}$, de 21 de setembro de 2017 (BR). Aprova a Política Nacional de Atenção Básica, estabelecendo a revisão de diretrizes para a organização da Atenção Básica, no âmbito do Sistema Único de Saúde (SUS). Diário Oficial da União, Brasília (DF), 22 set 2017.

7. Silva NM, Santos MA, Rosado SR, Galvão CM, Sonobe HM. Psychological aspects of patients with intestinal stoma: integrative review. Rev Lat Am Enfermagem. 2017;25:e2950. http://dx.doi.org/10.1590/15188345.2231.2950. PMid:29236836.

8. Simon BS, Schimith MD, Silveira CL, Budó MLD, Silva MEN, Garcia RP. Configuração da rede de assistência às pessoas com estomia: interface do cuidado continuado. J Nutr Health [Internet]. 2014; [citado 2018 dez 1];4(1):65-76. Disponível em: https://periodicos.ufpel.edu.br/ ojs2/index.php/enfermagem/article/view/3394/3509

9. Minayo MCS, organizador. Pesquisa social: teoria, método e criatividade. 29. ed. Petrópolis: Vozes; 2016.
10. Resolução $n^{\circ}$ 466, de 12 de dezembro de 2012 (BR). Dispõe sobre a pesquisa com seres humanos. Diário Oficial da União, Brasília (DF), 13 dez 2012.

11. Rodrigues RV. A importância das orientações de enfermagem para a adaptação dos pacientes com estomia intestinal [monografia]. Santa Cruz do Sul: Universidade de Santa Cruz do Sul; 2016.

12. Sá DLF, Pinheiro RX, Araújo MMO, Falcão PC, Chagas MX. Cuidados com o paciente pré-cirúrgico de ostomia. Rev Cient Multi Núcleo Conhece[Internet]. 2018; [citado 2018 dez 1];4(1):21-30. Disponível em: https://www.nucleodoconhecimento.com.br/saude/paciente-precirurgico-de-ostomia

13. Castro ABS, Benício CDAV, Carvalho DC, Monte NF, Luz MHBA. Conhecimentos e práticas de pessoas estomizadas: um subsídio para o cuidar em enfermagem. Rev Estima. 2014;12(4):21-28.

14. Calmon DPL. Elaboração de protocolo de assistência de enfermagem ao paciente colostomizado [monografia]. Vitória: Faculdade Católica Selesiana do Espírito Santo; 2014 [citado 2018 dez 1]. Disponível em: http://www.ucv.edu.br/fotos/files/TCC_2014-2_Dayana.pdf

15. Schwartz MP, Sá SPC. Ação educativa do enfermeiro no pré-operatório da confecção do estoma intestinal: revisão integrativa. Rev. Enferm. UFPE 2013 out; $7(10): 6233-7$

16. Anjos Fo NC, Souza AMP. A percepção sobre o trabalho em equipe multiprofissional dos trabalhadores de um centro de atenção psicossocial em Salvador, Bahia, Brasil. Interface. 2016 mar;21(60):63-76. http:// dx.doi.org/10.1590/1807-57622015.0428.

17. Moreira LR, Souza JC, Oliveira MM, Melo NS, Cerqueira TF. Autocuidado com estomias: compreensão de pacientes hospitalizados acerca das orientações recebidas pela equipe. Rev Enferm. 2017; [citado 2018 dez 1];20(2):1-19 Disponível em: http://periodicos.pucminas.br/index.php/ enfermagemrevista/article/view/16329

18. Marques GS, Nascimento DC, Rodrigues FR, Lima CMF, Jesus DF. A vivência de pessoas com estomia intestinal no grupo de apoio em um Hospital Universitário. Rev. Hosp. Univ. Pedro Ernesto. 2016;15(2):11321. http://dx.doi.org/10.12957/rhupe.2016.28235.

19. Pereira CR. Construção e validação de uma cartilha de orientação sobre o tratamento quimioterápico [dissertação]. Fortaleza: Programa de Pós-graduação em Políticas Públicas e Gestão da Educação Superior, Universidade Federal do Ceará; 2014 [citado 2018 dez 1]. Disponível em: http://www.repositorio.ufc.br/handle/riufc/8580

20. Poggetto MTD, Zufii FB, Luiz RB, Costa SP. Conhecimento do profissiona enfermeiro sobre ileostomia na Atenção Básica. Rev. Min. Enferm [Internet]. 2012; [citado $2018 \mathrm{dez}$ 1];16(4):502-8. Disponível em: http:// www.reme.org.br/artigo/detalhes/554

21. Santos CRS, Corrêa ACS, Silva D. Programa de Estratégia Saúde da Família sobre estomias intestinais e urinárias. Estima. 2017;15(2):161-8. http://dx.doi.org/10.5327/Z1806-3144201700030007.

22. Portaria ํㅜ 400 de 16 de novembro de 2009 (BR). Estabelecer Diretrizes Nacionais para a Atenção à Saúde das Pessoas Ostomizadas no âmbito do Sistema Único de Saúde -SUS, a serem observadas em todas as unidades federadas, respeitadas as competências das três esferas de gestão. Diário Oficial da União, Brasília (DF), 16 nov 2009.

23. Moura DBS. Assistência de enfermagem a pacientes estomizados no Brasil [monografia]. Tio Hugo: Universidade Federal de Santa Maria; 2011 [citado 2018 dez 1]. Disponível em: https://repositorio.ufsm.br/ bitstream/handle/1/2550/Moura_Daiane_Bianchessi_dos_Santos. pdf? sequence $=1$

24. Queiroz CG. Evidências de validade de instrumentos para coletas de dados no pré e pós-operatório de estomizados intestinais a luz do modelo de adaptação de Roy [dissertação]. Natal: Universidade Federa do Norte; 2017 [citado 2018 dez 1]. Disponível em: https://repositorio. ufrn.br/jspui/handle/123456789/24741

25. Moraes JT, Amaral CFS, Borges EL, Ribeiro MS, Guimarães EAA Serviços de atenção ao estomizado: análise diagnóstica no Estado de Minas Gerais, Brasil. Cad Saude Colet. 2014 mar;22(1):101-8. http:// dx.doi.org/10.1590/1414-462X201400010015.

26. Barba PD, Bittencourt VLL, Kolankiewicz ACB, Loro MM. Demandas de cuidados de pacientes oncológicos estomizados assistidos na atenção primária à saúde. Rev Enferm UFPE. 2017;11(8):3122-9. 